ACCEPTED MANUSCRIPT

\title{
Impulsive generation of $<100>$ dislocation loops in BCC iron
}

To cite this article before publication: Andres Bertoni et al 2020 Modelling Simul. Mater. Sci. Eng. in press https://doi.org/10.1088/1361$\underline{651 X / a b 81 a 7}$

\section{Manuscript version: Accepted Manuscript}

Accepted Manuscript is "the version of the article accepted for publication including all changes made as a result of the peer review process, and which may also include the addition to the article by IOP Publishing of a header, an article ID, a cover sheet and/or an 'Accepted Manuscript' watermark, but excluding any other editing, typesetting or other changes made by IOP Publishing and/or its licensors"

This Accepted Manuscript is ( 2020 IOP Publishing Ltd.

During the embargo period (the 12 month period from the publication of the Version of Record of this article), the Accepted Manuscript is fully protected by copyright and cannot be reused or reposted elsewhere.

As the Version of Record of this article is going to be / has been published on a subscription basis, this Accepted Manuscript is available for reuse under a CC BY-NC-ND 3.0 licence after the 12 month embargo period.

After the embargo period, everyone is permitted to use copy and redistribute this article for non-commercial purposes only, provided that they adhere to all the terms of the licence https://creativecommons.org/licences/by-nc-nd/3.0

Although reasonable endeavours have been taken to obtain all necessary permissions from third parties to include their copyrighted content within this article, their full citation and copyright line may not be present in this Accepted Manuscript version. Before using any content from this article, please refer to the Version of Record on IOPscience once published for full citation and copyright details, as permissions will likely be required. All third party content is fully copyright protected, unless specifically stated otherwise in the figure caption in the Version of Record.

View the article online for updates and enhancements. 


\title{
Impulsive generation of $<\mathbf{1 0 0}>$ dislocation loops in $\mathrm{BCC}$ iron
}

\author{
A.I. Bertoni ${ }^{1,2}$, , O.R. Deluigi ${ }^{2,3}$, G.J. Dos Santos ${ }^{2,3}$, M. Perez Díaz ${ }^{1}$, E.M. Bringa ${ }^{2,3,4 *}$ \\ *Corresponding author; e-mail: ebringa@yahoo.com \\ ${ }^{1}$ Instituto Interdisciplinario de Ciencias Básicas (ICB-CONICET), Universidad Nacional de Cuyo, \\ Mendoza 5502, Argentina \\ ${ }^{2}$ CONICET, Mendoza, Argentina \\ ${ }^{3}$ Facultad de Ingeniería, Universidad de Mendoza, Mendoza 5500, Argentina \\ ${ }^{4}$ Centro de Nanotecnología Aplicada, Facultad de Ciencias, Universidad Mayor, Chile
}

\begin{abstract}
The conditions for the formation of $<100>$ dislocation loops in $\mathrm{BCC}$ iron were investigated via Molecular Dynamics simulations using a simplified model intended to mimic conditions in high energy collision cascades, focusing on the possible coherent displacement of atoms at the boundary of a subcascade. We report on the formation of $<100>$ dislocation loops due to the fast displacement of a few hundred atoms with a coherent acceleration, in agreement with previous results for much larger cascade simulations. We analyze in detail the resulting atomic velocities and pressures, and find that they cannot be described within the usual formalism for a shock regime, since the pressure pulse only lasts less than 1 ps and does not match expected values from a Hugoniot shock. Our simulations include two interatomic potentials: Mendelev, which is extensively used for radiation damage simulations, and Ackland, which has been used for shock simulations because it can reproduce the experimentally observed transition from $\mathrm{BCC}$ to $\mathrm{HCP}$ structure at around $25 \mathrm{GPa}$, at high deformation rates. They both show similar evolution of defects, also indicating departure from a shock regime which is extremely different for these potentials.
\end{abstract}

\section{Introduction}

Iron allotropes in a body-centered cubic (BCC) crystalline phase take part in industrial processes worldwide as many steels: materials that display remarkable thermo-mechanical properties. Iron plays an important role as a structural material in nuclear reactors and the generation of radiation-induced defects has to be understood in order to predict reactor lifetime [Stoller 2012, Nordlund et al. 2018a]. Therefore, iron has inspired an enormous quantity of scientific studies, mainly on its mechanical and electromagnetic properties. However, some aspects of iron remain elusive for the moment.

The study of dislocations in BCC iron is important since they contribute to its unique hardness [Arakawa et al. 2007, Proville et al. 2012, He et al. 2017]. Early stages of radiation damage are often modeled with molecular dynamics simulations [Nordlund et al. 2018b] and show 
generation of $1 / 2<111>$ dislocation loops [Stoller 2012]. These radiation-produced dislocation loops are known to affect different properties of the materials, for example the yield stress of iron [Lehtinen et al. 2018] among others. Despite being generally accepted that the formation energy of $<100>$ dislocation loops is higher than that for $<111>$ loops [Marian et al. 2002], experiments observe mostly $<100>$ loops using transmission electron microscopy (TEM) [Carrington et al. 1960, Masters 1963, Eyre and Bartlett 1964, Masters 1965, Dudarev et al. 2008, Yao et al. 2010, Schäublin et al. 2017]. The formation mechanism for these $<100>$ dislocation loops is still under study with no completely satisfactory explanation given so far. There are diffusive mechanisms for the formation of $<100>$ dislocation loops in BCC iron, which require simulation time lengths much longer than those in typical MD simulations of primary damage. To explore these time scales, some efforts have been made using both MD [Chen et al. 2013, Gao et al. 2017, Wang et al. 2019a, Wang et al. 2019b] and Kinetic Monte Carlo simulations [Xu et al. 2013a, Xu et al. 2013b]. Formation due to overlap of cascades with pre-existing defects has also been reported [Byggmästar et al. 2019]. Irradiation by swift heavy ions can also lead to $<100>$ loop production [Khara et al. 2017].

As an alternative to diffusion mechanisms for the formation of $<100>$ loops, direct formation during the cascade lifetime has been recently reported. Sand et al. observed one event of formation on the impact of a $150 \mathrm{keV}$ tungsten primary knock-on atom (PKA) as a direct result of the cascade collapse [Sand et al. 2014].

A recent study in BCC iron [Peng et al. 2018] proposed a shock mechanism for the formation of $<100>$ loops. The described mechanism was reported to be very fast, i. e. of only a few picoseconds, and allegedly it consisted of a supersonic shockwave due to the displacement cascade. As reported, the stimulus of the supersonic shockwave would provide the necessary energy for the $<100>$ dislocation loops to nucleate directly from the lattice.

Shock physics can be described by the Hugoniot equations [Zel'Dovich et al. 1967], which gives an expression for the pressure of a steady state shock. Crystalline materials under pressure can undergo phase transformations; iron displays a transition from body-centered-cubic (BCC) to a hexagonal close-packed (HCP) structure at a pressure around $13 \mathrm{GPa}$, according to some early experiments [Boettger and Wallace 1997, Pepperhoff 2001, Smith et al. 2011]. Shocks in iron have been intensively studied using MD simulations. Kadau et al. used an embedded atom model (EAM) potential to uncover possible atomistic mechanisms for this transition [Kadau et al. 2002, Kadau et al. 2005]. However, the potential they used did not properly describe BCC iron plasticity. On the other hand, the Mendelev potential [Mendelev et al. 2003], often used in simulations of plasticity and radiation damage in iron, did not properly describe the high pressure phase transition, which was shifted to nearly $70 \mathrm{GPa}$. To solve this, Gunkelmann et al. presented a modification of the Mendelev potential, which was able to model both plasticity and the phase transition [Gunkelmann et al. 2012]. They found not only dislocation plasticity below the phase transition, but also that the transition mostly led to a HCP phase [Gunkelmann et al. 2014a]. For nanocrystalline iron they found a transition pressure near $25 \mathrm{GPa}$ [Gunkelmann et al. 2014b], which is consistent with the experimental results of Crowhurst et al. for ultra-short shocks, i. e. of tens of ps [Crowhurst et al. 2014]. 
There are several studies showing the role of pressure in defect formation due to ion irradiation. Ghaly and Averback performed molecular dynamics simulations of $10 \mathrm{keV}$ and $20 \mathrm{keV}$ gold bombardment of metallic substrates [Ghaly and Averback 1994]. The authors argued that high pressure is generated in the cascade core, due to the thermal spike, giving rise in some cases to the formation of cavities. These cavities increased the probability of defect formation in cascades by interstitial loop punching. However, pressures were below 10 $\mathrm{GPa}$, and their spatial profiles showed that its influence never reached beyond $3 \mathrm{~nm}$ from the cascade core, with a spike lifespan of about 1 ps. Soon after that publication, Ghaly et al. also reported flows of atoms arising from pressure created within molten cascade cores, for MD simulations of self-ion bombardment of several metals $(\mathrm{Au}, \mathrm{Cu}, \mathrm{Ge}, \mathrm{Pt})$ with ion energies in the range of 5-10 keV [Ghaly et al. 1995]. The presented pressure profiles around the vicinity of the cascade declined greatly after 0.5 ps. Nordlund et al. [Nordlund et al. 1999] showed from MD simulations of high-energy self-bombardment metallic bulk, that the high pressures developed in collision cascades can cause a coherent displacement of thousands of atoms in a shear-induced mechanism. Such collision cascades form a liquid-like region deep into the bulk, where the extreme heat produces a large compressive stress on the surrounding crystal, triggering the formation of interstitial dislocation loops and surface modification. Again, in this cited work, the pressure peak along the displacement direction ( $\mathrm{z}$ axis) was shown to last around 1 ps. Shock and impulse models have also been used for irradiation in the electronic regime to explain surface features [Papaleo ett al. 2015], DNA damage [Fraile et al. 2019], etc.

Using Molecular Dynamics (MD) simulations, we mimic the coherent fast displacement of a group of atoms, as observed in sub-cascades splitting from the main high-energy collision cascade which leads to formation of stable $<100>$ dislocation loops in BCC iron [Peng2018]. By using a simplified model, we significantly reduce both the time and the system size needed for this type of simulations. We investigate under which conditions these loops are formed, while characterizing pressure and velocity of the system and comparing our results with previous literature findings.

\section{Method}

Regular approaches for recreating the initial conditions of high-energy collision cascades require time lengths similar to those used in experiments, which often have to be modeled with other techniques like Kinetic Monte Carlo [Xu et al. 2013a, Xu et al. 2013b] or by modelling systems with a colossal amount of atoms [Peng et al. 2018]. Molecular Dynamics simulations of high-energy/collision cascades of more than $100 \mathrm{keV}$ typically require hundreds of millions of atoms and therefore can only be carried out in large supercomputer clusters.

In the present work, we performed MD simulations on BCC iron (lattice parameter $\mathrm{a}_{0}=$ 2.8553 A) using a simple model that manages to replicate the conditions from some displacement cascades caused by high-energy collision events. In the proposed model (see Figure 1), a sphere of heated atoms is generated inside the material to match the temperature 
of a hot cascade core. Then, a group of a few hundred atoms within a cylindrical region, located on the upper boundary of the hot sphere, is accelerated along the cylinder main axis to mimic the coherent displacement of atoms which can occur at the boundary of the cascade, as reported before [Ghaly and Averback 1994, Ghaly et al. 1995, Nordlund et al. 1999, Peng et al. 2018]. The simplicity of the model we propose to recreate the coherent displacement of atoms that arises from high-energy collision cascades, allow us to explore the formation of crystal defects with a reduced amount of atoms in very short times. Essentially, this approach tries to mimic only the front group of atoms of a sub-cascade that would result from the main high-energy collision cascade.
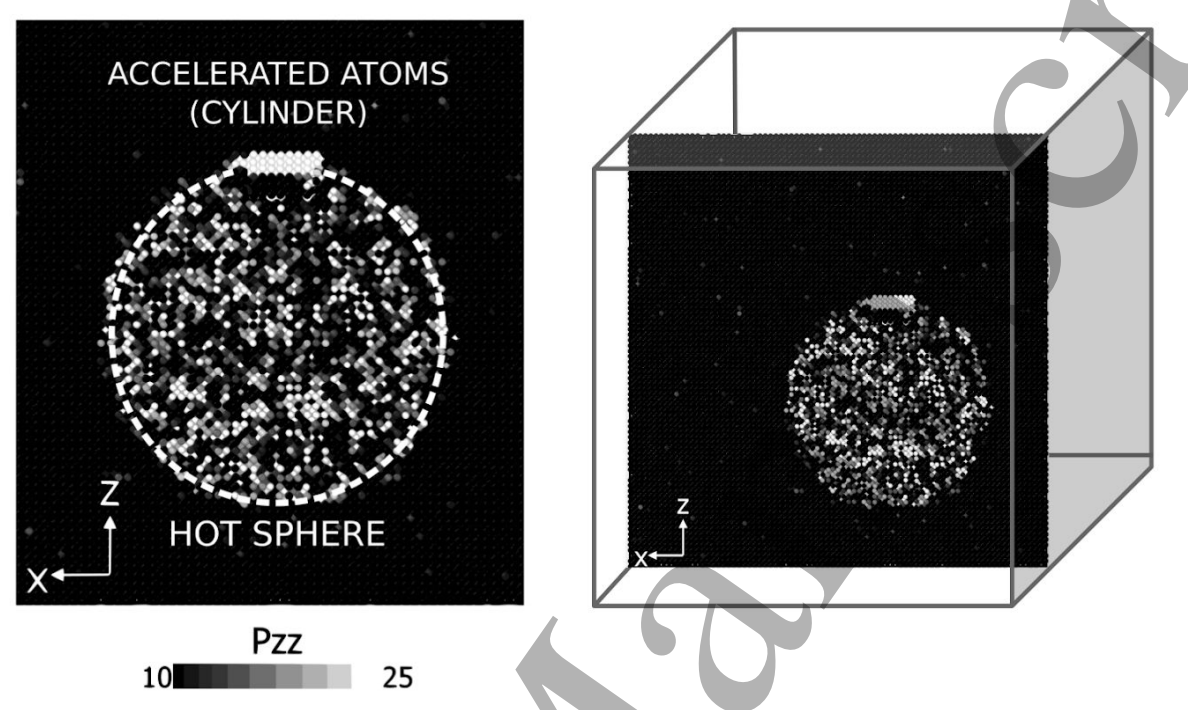

Figure 1 - Snapshot of a slice of the system along the xz plane. It corresponds to the state of the system at $0.1297 \mathrm{ps}$ and it's color coded according to the pressure along the $\mathrm{z}$ axis (between $10 \mathrm{GPa}$ and $25 \mathrm{GPa}$ ). On the left figure, the dashed line marks the boundaries of the hot sphere. On top of it, there is a group of high-pressure atoms that corresponds to the cylinder of accelerated atoms.

In our simulations, the interaction between atoms is computed using the EAM potential developed by Ackland and co-workers [Gunkelmann et al. 2012], which has been extensively tested and it is able to reproduce the BCC to HCP structural transition occurring at around 25 GPa [Gunkelmann et al 2014a, Gunkelmann et al 2014b]. In order to compare results, simulations were also run using the very popular EAM interatomic potential for radiation damage simulations, developed by Mendelev [Mendelev et al 2003].

In simulation studies of high-energy collision cascades, it is necessary to use short-range repulsive potentials to compute the short-distance interatomic interactions that may arise at early stages of these high energy events, which are common in radiation damage simulations. Typically, the ZBL [Ziegler et al 1985] potential is used for interatomic distances smaller than $\sim 0.1 \mathrm{~nm}$ and a hybrid potential (combining ZBL with another typical interatomic potential) for distances typically between $\sim 0.1 \mathrm{~nm}$ and $\sim 0.2 \mathrm{~nm}$. However, there is no general agreement on this potential switching, as discussed for instance in [Stoller 2016]. Nevertheless, since our model does not include the primary knock-on atom (PKA) nor the initial primary collisions, these short-distance interactions are highly infrequent. The Mendelev potential we employed already includes the ZBL potential at short distances. We have carried simulations 
for the Ackland potential reported in shock studies, but also for a hybrid ZBL-Ackland potential through an overlay function included in the employed simulation software; the inner cutoff was set at $0.1 \mathrm{~nm}$ and the outer cutoff at $0.2 \mathrm{~nm}$. As expected, given that we do not have high energy collisions, results for the Ackland and hybrid ZBL-Ackland do not display any significant difference. A plot of all these potentials is shown in Figure S6 of the supplementary material.

Simulations were carried out with the LAMMPS package [Plimpton 1995] and the resulting samples were visualized and analyzed with OVITO [Stukowski 2010]. We used several tools from this software to carry out the analysis, including "Dislocation eXtraction Algorithm" (DXA) [Stukowski et al 2012], "Polyhedral template matching" (PTM) [Larsen et al 2016], and Wigner-Seitz defect analysis.

The simulation cell consisted of a cubic box $\left(L_{x}=L_{y}=L_{z}\right.$ ) with edges of $80 \mathrm{a}_{0}$, holding 1,024,000 atoms, where the $x, y$ and $z$ axes were oriented along the [100], [010] and [001] directions, respectively. Periodic boundary conditions (PBC) were implemented on all three axes. The hot sphere had a radius of $20 \mathrm{a}_{0}$ and was centered at $(-10 ;-10 ;-10) \mathrm{a}_{0}$; the temperature of the atoms enclosed within this region was scaled up to $2500 \mathrm{~K}$. Another group of atoms within a cylindrical region located at the surface of the hot sphere was accelerated along the cylinder main axis, giving them an instantaneous initial velocity between $2 \mathrm{~km} / \mathrm{s}$ and $9 \mathrm{~km} / \mathrm{s}$. All cylinders had a radius of $4 \mathrm{a}_{0}$ with its main axis set parallel to the $\mathrm{z}$ axis (the [001] direction) and centred at $(-10,-10) a_{0}$ in the $x y$ plane. The centre of the top face of the cylinders was fixed at $\mathrm{z}=9 \mathrm{a}_{0}$, close to the upper edge of the hot sphere, while the centre of the lower face varied between $z=4 a_{0}$ and $z=7 a_{0}$ by $1 a_{0}$, giving a total of 4 cylindrical regions of different sizes, enclosing between 197 and 495 atoms.

After a preliminary energy minimization, including box relaxation, the system was held at 300 $\mathrm{K}$ and zero pressure, before the modeled/cascade phase took place. Given that this process involved large velocities and accelerations, a variable time step was used for about $2 \mathrm{ps,}$ followed by a simulation using 1 fs steps. Finally, in order to track the cascade evolution and test the stability of the radiation-induced defects, the system was simulated during additional 35 ps using a microcanonical ensemble (NVE).

\section{Results and discussion}

As a first step, for the sake of comparison with the results reported by Peng et al. [Peng et al. 2018], we have calculated the root mean square displacement (RMSD) along the $\mathrm{z}$ axis of the accelerated atoms and a root mean square velocity (RMSV) also along z calculated as the time derivative of the RMSD (since this is the way that was calculated in the aforementioned study). Figure 2 shows these two quantities as a function of time for a cylinder of height $4 \mathrm{a}_{0}$ with initial velocity of $5 \mathrm{~km} / \mathrm{s}$. The results obtained with our simpler model show a similar behavior to those reported by Peng et al. [Peng 2018] 


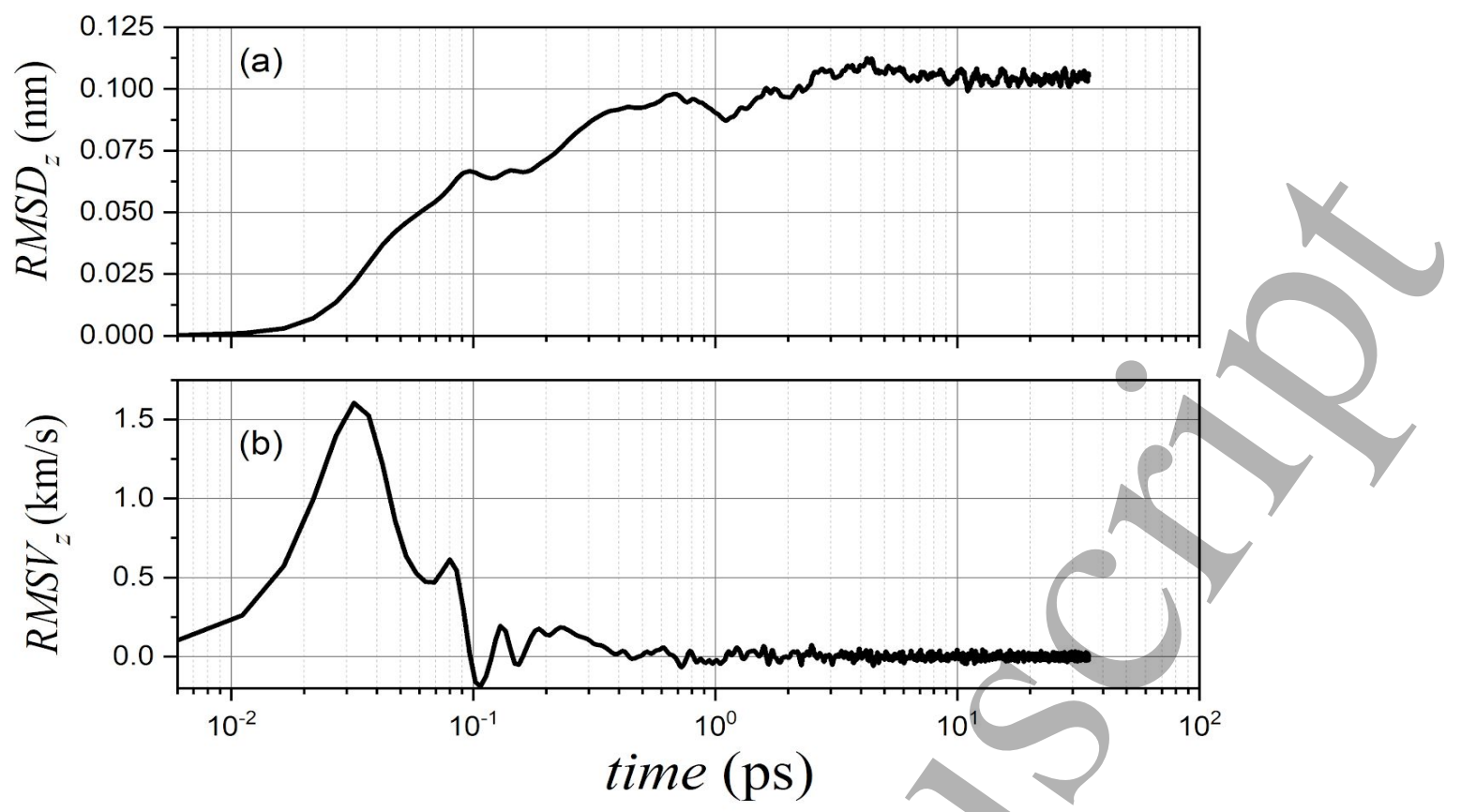

Figure 2 - Root mean square displacement (RMSD) along the $\mathrm{z}$ axis for the accelerated atoms $(a)$ and root mean square velocity along the $\mathrm{z}$ axis calculated as the time derivative of the RMSD $(b)$.

Beyond this initial comparison, we think that the most relevant quantities to report are the mean velocity and the kinetic energy per atom. While being accelerated, atoms within the cylindrical region showed per-atom mean kinetic energy profiles similar to those recently reported for groups of atoms with coherent displacement at the boundary of high-energy collision cascades [Peng et al. 2018], as can be seen in Figure 3. The curves regarding the mean velocity of the accelerated atoms also show time profiles with qualitatively the same shape as those reported in the mentioned study.
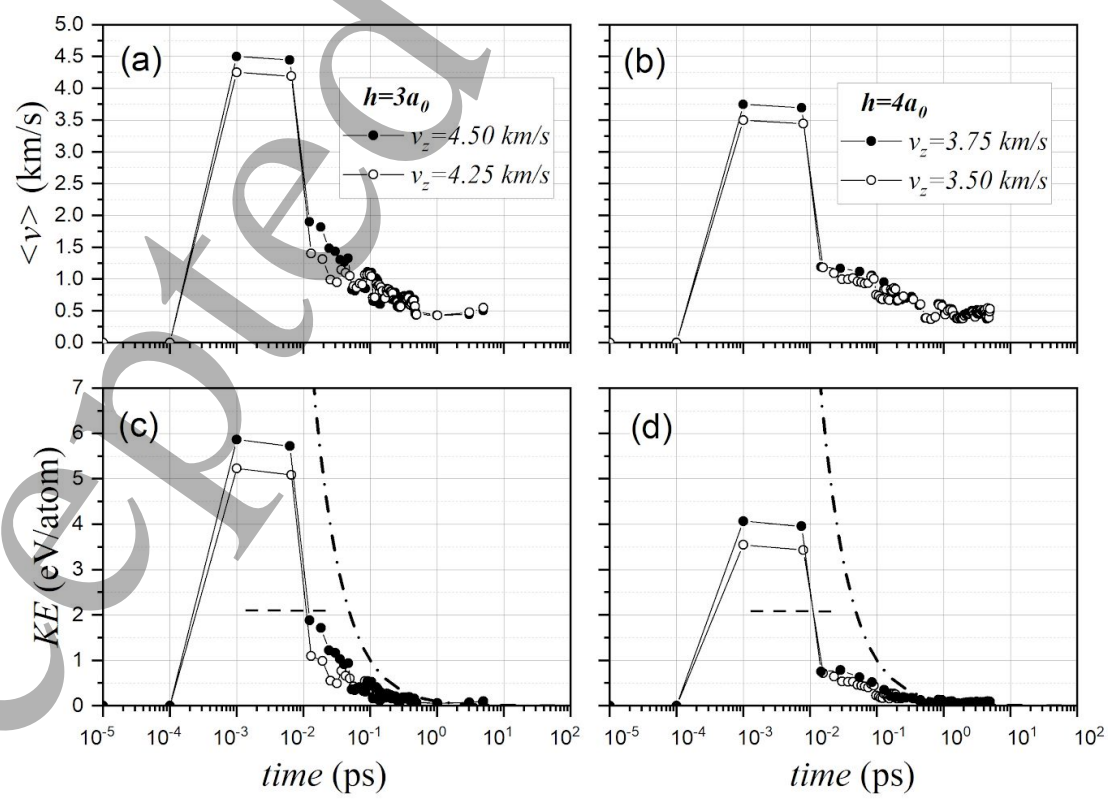

Figure 3 - The two upper graphs show the evolution of the mean velocity $(<v>)$ for the accelerated atoms within the cylinders of heights $3 \mathrm{a}_{0}(a)$ and $4 \mathrm{a}_{0}(b)$ (being $\mathrm{a}_{0}$ the lattice parameter). Displayed data corresponds to initial velocities immediately before and after the velocity threshold for the 
formation of $<100>$ dislocation loops (filled and void dots, respectively). Graphs on the bottom show the mean kinetic energy per atom $(K E)$ within the accelerated cylinders, for the cylinder of $h=3 a_{0}$ (c) and for the cylinder of $h=4 a_{0}(d)$. The dash-dotted curve represents a fit for the decaying part of the curves, proportional to $1 / \mathrm{t}$. The horizontal dashed line corresponds to the energy threshold reported in Fig. 2 from [Peng et al. 2018].

In Fig. 2 of [Peng et al. 2018], formation energies of about $2.2 \mathrm{eV}$ and $1.9 \mathrm{eV}$ are considered for $<100>$ and $<111>$ loops, respectively. From ab-initio simulations [Alexander et al. 2016], a loop with 100 self interstitial atoms (SIA) similar to the ones appearing in our simulations, requires about $1.4 \mathrm{eV} /$ atom for $<100>$ loops and $1.1 \mathrm{eV} /$ atom for $<111>$ loops. We note that, in our simulations, the maximum mean kinetic energy of the coherently displaced atoms is well above the formation energy of these loops, even for the cases where there is no observed loop formation.

For the cylinder of height $3 \mathrm{a}_{0}$, the kinetic energy threshold for the $<100>$ dislocation loop formation was found to be of $5.86 \mathrm{eV} /$ atom, while for the cylinder of height $4 \mathrm{a}_{0}$ that threshold was of $4.05 \mathrm{eV} /$ atom. It is notable that our simplified model is able to reproduce qualitatively the same results of large scale simulations with considerably less computational cost.

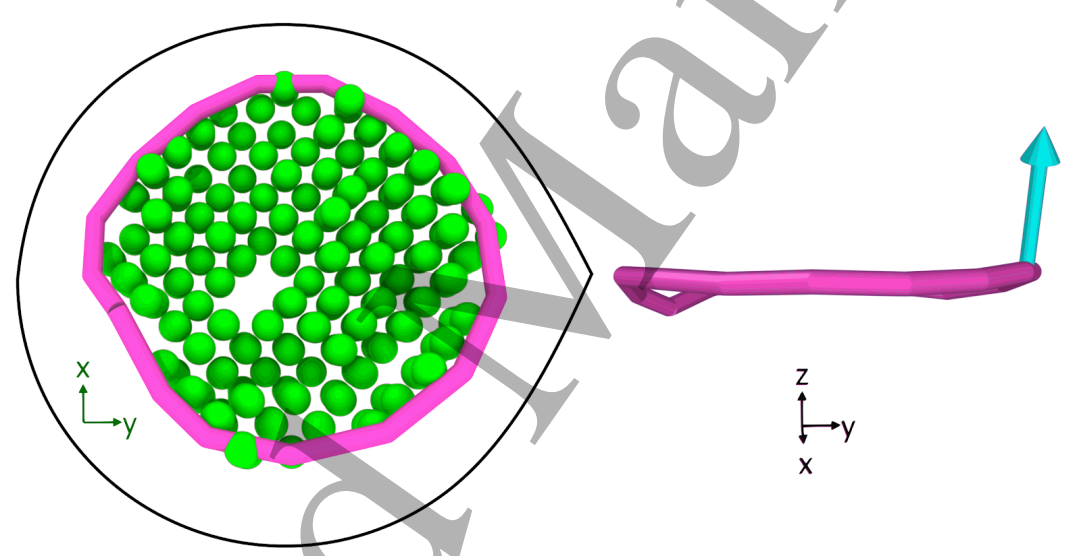

Figure 4 - Dislocation loops $<100>$ at the end of the simulation (after 35 ps in NVE conditions) for the case of the cylinder of height $4 \mathrm{a}_{0}$ with an initial velocity of $3.75 \mathrm{~km} / \mathrm{s}$. The inset figure shows a total of 101 interstitial atoms detected and isolated by a Wigner-Seitz (WS) defect analysis in the displaced configuration (occupancy of 2 in each WS cell). The arrow over the dislocation loop corresponds to its Burgers vector scaled by a factor of 3 .

Due to the coherent displacement of the accelerated atoms, the formation of $<100>$ dislocation loops was observed. Usually, a pair of dislocation loops was formed, one loop located near the starting point of the accelerated atoms (bottom loop) and the other one a bit further away in the direction of the positive z-axis (upper loop). A graphical representation of the coherent displacement along the $\mathrm{z}$ axis of the accelerated atoms and the loop formation process can be seen in Figure S1 (in the supplementary material), where the displacement vectors for the atoms clearly indicate the role of this process on loop formation. 
The upper dislocation loop contained self-interstitial atoms (see Figure 4), while the loop closer to the starting position of the cylinder contained vacancies. These loops were found to be stable after 35 ps of simulation in NVE conditions. These dislocations were identified as edge dislocations by DXA [Stukowski et al. 2012]. In a few cases, we observed the formation of $<100>$ dislocation loops containing some $<111>$ segments. However, considering that our simulations only lasted $35 \mathrm{ps,}$ we annealed one of these complex loops. After a few picoseconds at a temperature near $600 \mathrm{~K}$, the loop transformed into a pure $<100>$ loop, with a dislocation length variation of about $10 \%$ (see Figure S2 in the supplementary material). A fraction of a picosecond after the acceleration event, we have observed the formation of voids in the vicinity of the region of the cylinders, similar to those reported by Ghaly et al. [Ghaly and Averback 1994]. Snapshots of these events for both interatomic potentials are shown in Figure S3 in the supplementary material. The final temperature of our simulations was around $370 \mathrm{~K}$. This corresponds with our systems being large enough to successfully dissipate the temperature of the hot sphere.

The formation of stable $<100>$ dislocation loops was observed for a group of simulations. We found that, for a given height of the accelerated cylinder, such events depend on the initial velocity of the driven atoms. There is a velocity threshold, surprisingly well defined, above which the formation of dislocations loops is observed, as Figure 5 shows for the cylinder heights under study: the total $<100>$ dislocation length is plotted as a function of the initial velocity for the accelerated atoms of each cylinder. It can be seen that, above all thresholds, the dislocation length is of the order of the cylinder circular cross section perimeter (dashed line in Fig. 5), multiplied by two in order to account for the presence of two loops. The characteristics, orientation and number of loops found in each simulation are summarized in Table S1 reported in the supplementary material.

As can be seen, the velocity threshold for the $<100>$ dislocation loop formation decreases as the height of the cylinder increases. This relates to larger cylinders having more total linear momentum along the direction of acceleration than cylinders composed of fewer atom layers. When the upper atoms begin to decelerate due to collisions with atoms above the cylinder, the atoms behind continue to push them further in the displacement direction. 


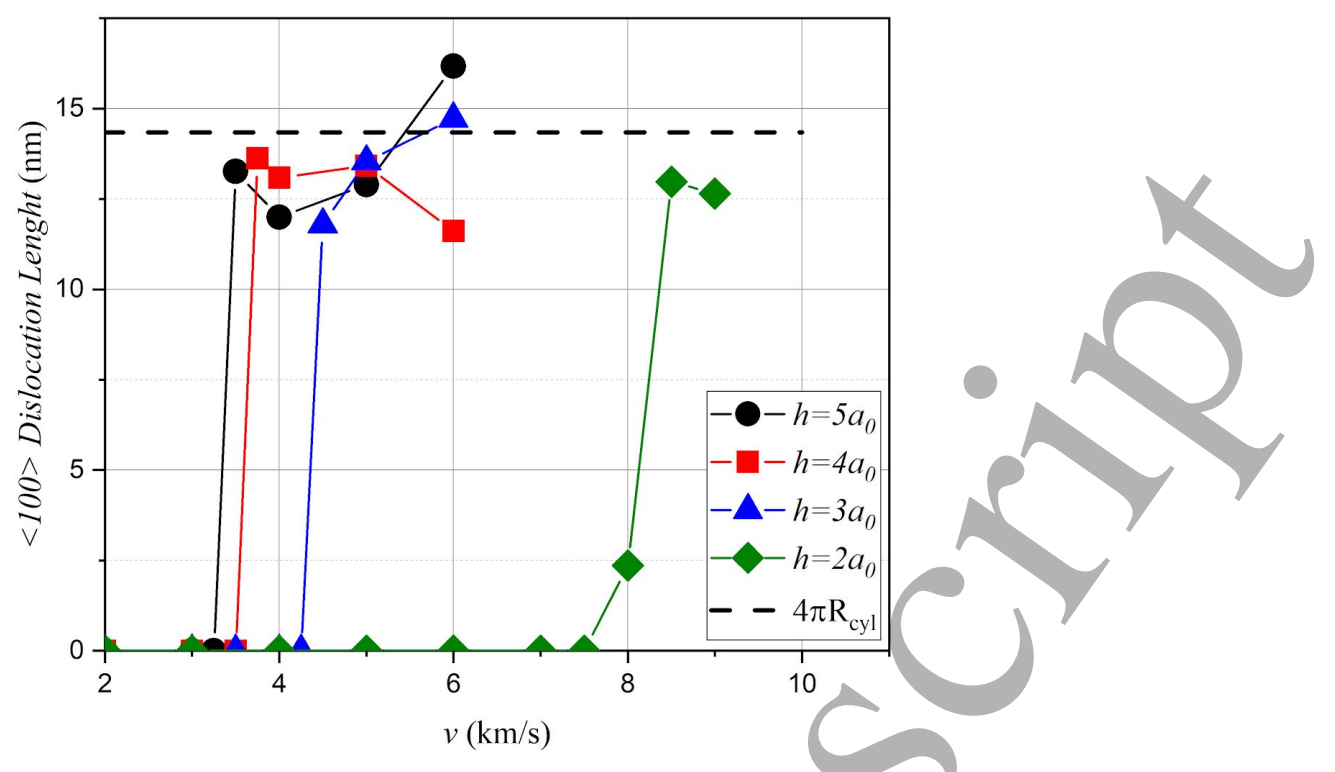

Figure 5 - Total $<100>$ dislocation length as a function of the initial velocity of the accelerated atoms for cylinders of different height $h$. The dashed line corresponds to twice the cylinder circular cross section perimeter (with a radius $\mathrm{R}_{\text {cyl }}$ of $4 \mathrm{a}_{0}$ ).

As mentioned before, high-energy collision cascades generates a localized increase of pressure along the direction of propagation of the wave [Ghaly and Averback 1994, Ghaly et al. 1995, Nordlund et al. 1999]. Figure 6 shows spatial pressure and velocity profiles along the acceleration direction ( $\mathrm{z}$ axis) for the case of the cylinder with height $4 \mathrm{a}_{0}$ and radius $\mathrm{R}_{\text {cyl }}$, averaging over all atoms in the cylindrical volume. Several profiles are shown to depict the time evolution of these quantities after the atoms in the cylindrical region were accelerated to an initial velocity of $5 \mathrm{~km} / \mathrm{s}$. This figure also shows that the peak pressure for the cylinder atoms last less than $1 \mathrm{ps}$, a pressure peak lifespan that matches previously reported values [Ghaly and Averback 1994, Ghaly et al. 1995, Nordlund et al. 1999,Korchuganov et al 2015]. It can also be observed that the increase of pressure is highly localized in accordance with previous results in the literature. In addition, we have conducted volumetric strain analyzes and the results are also in this direction, showing a very localized deformation near the zone of the accelerated atoms (Figure S4 in the supplementary material). Korchuganov et al [Korchuganov et al 2015] reported shock waves for PKAs in Fe. For [001] directed PKAs there are high pressures lasting about $0.1 \mathrm{ps}$. There is an extremely narrow velocity front, which decays rapidly with distance from the PKA. These results are consistent with our findings. 

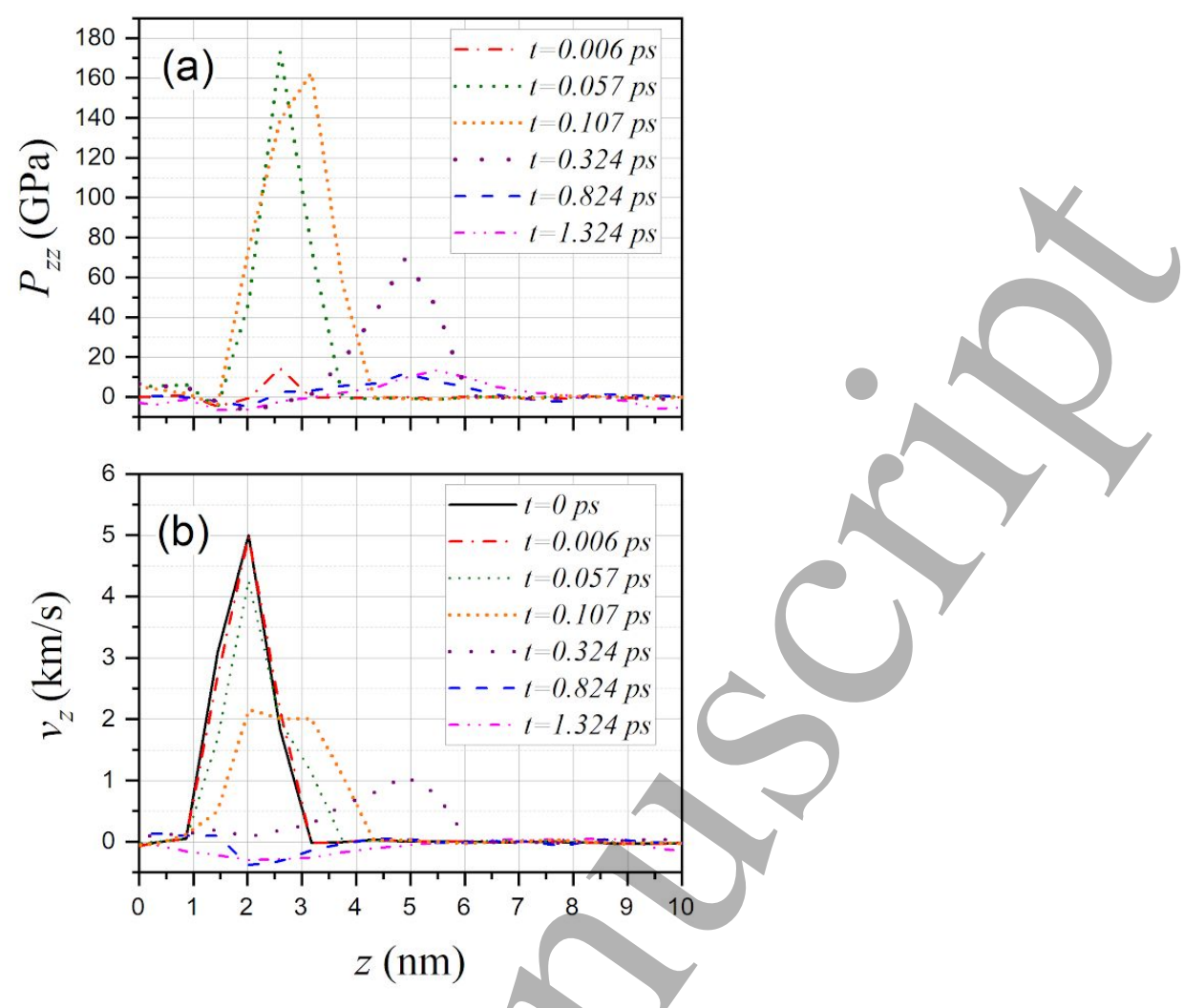

Figure 6 - Pressure $(a)$ and velocity $(b)$ spatial profiles along the acceleration direction (z axis) for the cylinder of height $4 \mathrm{a}_{0}$. Data is shown for a few moments after the atoms in the cylindrical region were accelerated to an initial velocity of $5 \mathrm{~km} / \mathrm{s}$.

Taking the Debye temperature $\left(\Theta_{D}\right)$ of iron as 470K [Kittel 2004], the Debye frequency was computed as $\omega_{D}=9.7910^{12} / \mathrm{s}$ with a corresponding period of $\tau_{D}=0.102 \mathrm{ps}$. By combined analysis of the atomic velocities and pressures (Fig. 6), we found that the impulsive effect which leads to the formation of $<100>$ dislocation loops only lasts about $0.5 \mathrm{ps}$, a time length which is of the order of the Debye period for iron and two orders of magnitude shorter than what is considered for ultra-short shock waves [Kanel et al. 2016].

Figure 7 shows that the simulation reaches pressures well above the phase transition pressure for the Ackland and the Mendelev potential (about $13 \mathrm{GPa}$ and $70 \mathrm{GPa}$, respectively [Gunkelmann et al. 2012]), but again this is only for a very short time of about $0.5 \mathrm{ps,} \mathrm{which}$ is insufficient for the defect formation to happen under thermodynamic conditions. As can be seen, this high pressure leads to approximately the same number of FCC $+\mathrm{HCP}$ atoms for both potentials, despite their very different transformation pressure thresholds, also indicating that the presence of these atoms is not related to a BCC to HCP shock transition. 


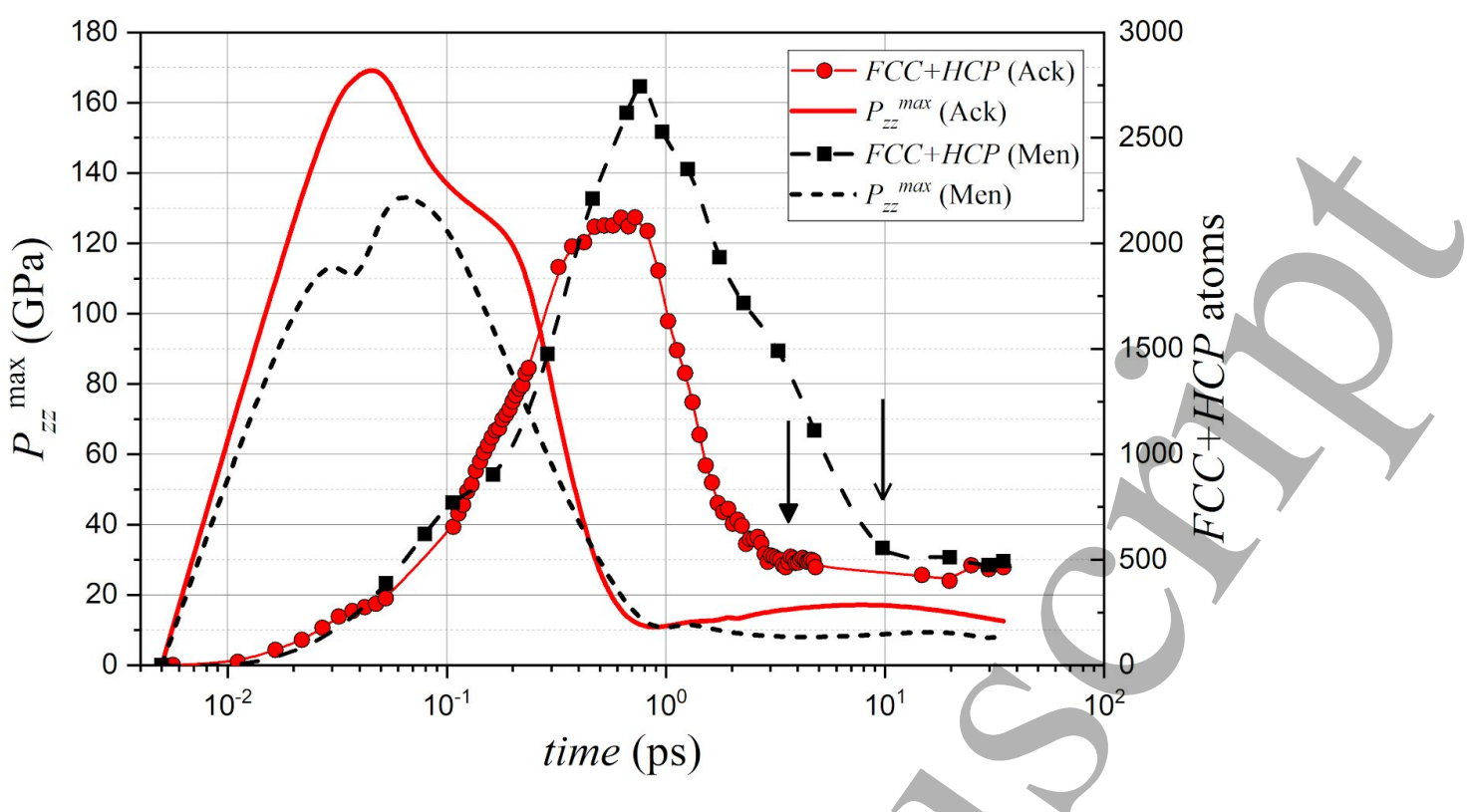

Figure 7 - Maximum pressure along the $\mathrm{z}$ axis (line plot) and number of $\mathrm{FCC}+\mathrm{HCP}$ atoms (line and symbol plot) over time for a cylinder of height $4 \mathrm{a}_{0}$ ( 8 layers of atoms) with an initial velocity of 5.0 $\mathrm{km} / \mathrm{s}$. These quantities were determined by simulations with two different interatomic potentials: Ackland [Gunkelmann et al. 2012] and Mendelev [Mendelev et al. 2003]. The arrows indicate when the formation of the $<100>$ dislocation loops occurred for both potentials.

For the atoms within the cylinder of height $4 \mathrm{a}_{0}$ and initial velocity of $5 \mathrm{~km} / \mathrm{s}$, we computed the atomic pressure over time, using both interatomic potentials. The time profiles for the atomic pressure are plotted in Figure 8, where we also compare them with the pressure expected for a shockwave according to the linear Hugoniot equations, $U_{s}=\left(c_{s}+s . U_{p}\right)$ and $P_{H}=U_{p} \cdot \rho . U_{s}$ [Zel'Dovich et al. 1967]. In these equations, $U_{p}$ is the velocity of a particle along the $\mathrm{z}$ axis, $c_{S}$ is the bulk speed of sound in iron, $s$ is the slope of Hugoniot, $\rho$ is the density of iron and $P_{H}$ the pressure of the shockwave. We have used the parameters reported in [Brown et al. 2000]. As the figure shows, for times under the Debye period $\left(\tau_{D}\right)$, using either potential, the pressures obtained via MD are much lower than the theoretical pressures for a shockwaye. Conversely, for either potential, pressures for $0.9 \mathrm{ps}$ after a Debye period are below the threshold pressure for the nanocrystalline iron BCC to HCP phase transition. We note that the transition pressure is strain-rate dependent, due to kinetic effects, reaching around $25 \mathrm{GPa}$ for high strain rates [Gunkelmann et al. 2014b, Crowhurst et al. $2014]$.

We have also carried out simulations using an overlay hybrid potential ZBL+Ackland [Gunkelmann et al 2012, Ziegler et al 1985]. After conducting the same analysis of Figures 6, 7 and 8 with the mentioned hybrid potential, we found that there is virtually no difference between the two approaches (as can be seen in figures S7, S8 and S9 at the supplementary material). We have also observed the production of stable dislocation loops of the same type and loop length. We believe this is a virtue of our simple model, which makes short-distance interactions highly infrequent, since it only mimics the front atoms of a sub-cascade that 
would result from the main high-energy collision cascade, allowing significantly smaller computational cost.

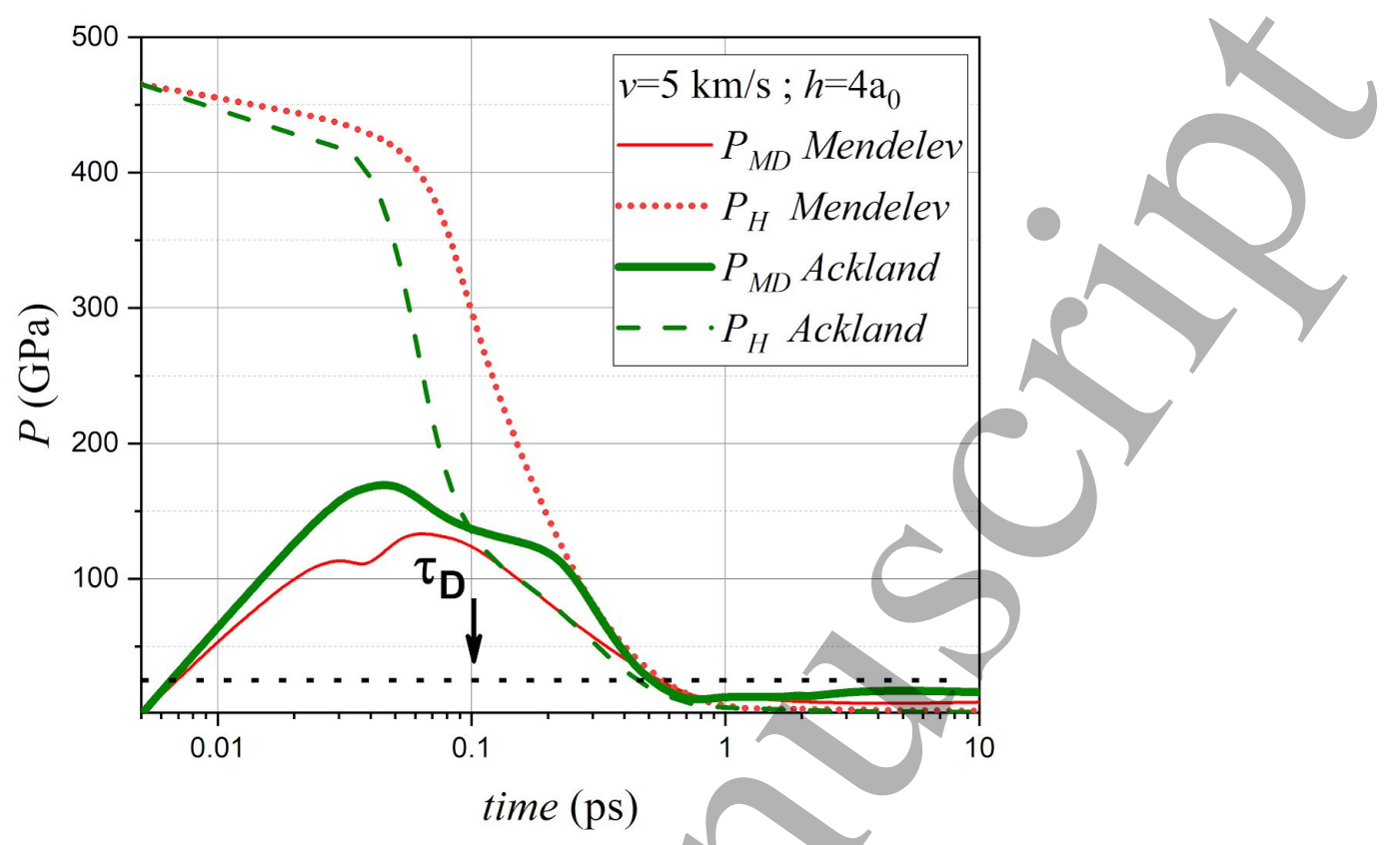

Figure 8 - Atomic pressure over time for the accelerated atoms of a cylinder of height $4 \mathrm{a}_{0}$ with an initial velocity of $5 \mathrm{~km} / \mathrm{s}$, obtained by Molecular/Dynamics simulation with the Mendelev [Mendelev et al. 2003] and Ackland [Gunkelmann et al. 2012] interatomic potentials. The dashed and dotted curves corresponds to the theoretical pressure of a shockwave, computed according to the Hugoniot equations [Zel'Dovich et al. 1967] $\left(P_{H}\right)$, while the short-dashed straight line is the threshold pressure for the nanocrystalline iron BCC to HCP phase transition (at $25 \mathrm{GPa}$ ) [Gunkelmann et al. 2014b, Crowhurst et al. 2014]. The down arrow corresponds to the Debye period ( $\left.\tau_{D}\right)$.

\section{Summary and conclusions}

Using a simplified model that avoids/replicating the initial conditions of high-energy collisions, we have presented evidence on the possible formation mechanism of $<100>$ loops during the early stages of energetic cascades, due to the fast displacement of a few hundred atoms with a coherent acceleration; all in agreement with previous results for much larger cascade simulations. By analysis of the resulting atomic velocities and pressures, we found that the impulsive effect which leads to the formation of dislocation loops only lasts about 0.5 ps: a time span which is of the order of the Debye period for iron and two orders of magnitude shorter than what is typically, considered for ultra-short shock waves. In the loop formation process, there is not enough time for a scenario comparable to the thermodynamic shock described by the Hugoniot equations. The atomic pressures in our simulations are significantly lower than those which would be achieved under shock conditions. Radiation simulations which might include high pressure shock conditions should be carried out with interatomic potentials which are able to reproduce shock results, alongside the defect energetic relevant to radiation-induced defect evolution. Our simulations with two different interatomic potentials (Mendelev [Mendelev et al. 2003] and Ackland [Gunkelmann et al. 2012]) showed similar evolution of defects, despite the fact that they behave very differently 
under shock conditions. Based on our modeled cascade simulations, a dislocation loop formation process due to fast coherent atomic displacement does not appear to be related to a high pressure shock regime.

\section{Acknowledgements}

This work used the Toko cluster from FCEN-UNCuyo, which is part of the SNCAD-MinCyT, Argentina. We thank helpful comments by A. Caro and H.J. Urbassek.

\section{Author's contributions}

$\mathrm{AB}, \mathrm{OD}, \mathrm{GD}$ run and analyzed simulations. MPD run simulations. EMB designed the simulations and contributed to the analysis. All authors contributed to the text.

\section{References}

- [Alexander et al. 2016] - Alexander, R.; Marinica, M. C.; Proville, L.; Willaime, F.; Arakawa, K.; Gilbert, M.R. and Dudarev, S.L. "Ab initio scaling laws for the formation energy of nanosized interstitial defect clusters in iron, tungsten, and vanadium"; Physical Review B 94 (2), 024103. (2016)

- [Arakawa et al. 2007] - Arakawa, K.; Ono, K.; Isshiki, M.; Mimura, K.; Uchikoshi, M. and Mori, H. "Observation of the one-dimensional diffusion of nanometer-sized dislocation loops"; Science 318 (5852), pp 956-959. (2007)

- [Boettger and Wallace 1997] - Boettger, J. C. and Wallace, D. C. "Metastability and dynamics of the shock-induced phase transition in iron"; Physical Review B 55 (5), 2840. (1997)

- [Brown et al. 2000] - Brown, J. M., J. N, Fritz, and R. S. Hixson. "Hugoniot data for iron"; Journal of Applied Physics 88 (9), pp. 5496-5498. (2000)

- [Byggmästar et al. 2019] - Byggmästar, J.; Granberg, F.; Sand, A.E.; Pirttikoski, A.; Alexander, R.; Marinica, M.C. and Nordlund, K. "Collision cascades overlapping with self-interstitial defect clusters in Fe and W." Journal of Physics: Condensed Matter 31 (24), 245402. (2019)

- [Carrington et al.1960] - Carrington, W.; Hale, K. F. and McLean, D. “Arrangement of dislocations in iron"; Proceedings of the Royal Society of London 259 (1297), pp 203-227. (1960)

- [Chen et al. 2013]-Chen, J.; Gao, N.; Jung, P. and Sauvage, T. "A new mechanism of loop formation and transformation in BCC iron without dislocation reaction"; Journal of Nuclear Materials 441 (1-3), pp. 216-221. (2013)

- [Crowhurst et al. 2014] - Crowhurst, J. C.; et al. "The $\alpha \rightarrow \epsilon$ phase transition in iron at strain rates up to $109 \mathrm{~s}-1$ "; Journal of Applied Physics 115 (11), 113506. (2014)

- [Dudarev et al. 2008] - Dudarev, S. L.; Bullough, R.; Derlet, P. M. "Effect of the $\alpha-\gamma$ phase transition on the stability of dislocation loops in BCC iron"; Physical Review Letters 100 (13), pp 135503. (2008) 
- [Eyre and Bartlett 1964] - Eyre, B. L. and Bartlett, A. F. "An electron microscope study of neutron irradiation damage in alpha-iron"; The Philosophical Magazine 12 (116), pp 261-272. (1964)

- [Fraile et al. 2019] - Fraile, A.; Smyth, M.; Kohanoff, J. and Solov'yov, A. V. "First principles simulation of damage to solvated nucleotides due to shock waves"; The Journal of Chemical Physics 150 (1), 015101. (2019)

- [Gao et al. 2017] - Gao, N; Chen, J; Kurtz, R. J.; Wang, Z.G.; Zhang, R.F. and Gao, F. "New understanding of nano-scale interstitial dislocation loops in BCC iron"; Journal of Physics: Condensed Matter 29 (45), 455301. (2017)

- [Ghaly and Averback 1994] - Ghaly, M. and Averback, R. S. "Effect of viscous flow on ion damage near solid surfaces"; Physical Review Letters 72 (3), pp. 364-367. (1994)

- [Ghaly et al. 1995] - Ghaly, M.; Averback, R. S. and De La Rubia, T. D. "Surface effects on damage production during ion bombardment: A molecular dynamics study"; Nuclear Instruments and Methods in Physics Research Section B 102 (1-4), pp. 51-57. (1995)

- [Gunkelmann et al. 2012] - Gunkelmann, N.; Bringa, E.M.; Kang, K.; Ackland, G.J.; Ruestes, C.J. and Urbassek, H.M., "Polycrystalline iron under compression: plasticity and phase transitions"; Physical Review B 86 (14), pp 144111. (2012)

- [Gunkelmann et al. 2014a] - Gunkelmann, N.; Bringa, E.M.; Tramontina, D.R.; Ruestes, C.J.; Suggit, M.J.; Higginbotham, A.; Wark, J.S. and Urbassek, H.M. "Shock waves in polycristalline iron: Plasticity and phase transitions"; Physical Review B 89 (14), 140102(R). (2014)

- [Gunkelmann et al. 2014b] - Gunkelmann, N.; Tramontina, D.R.; Bringa, E.M. and Urbassek, H.M. "Interplay of plasticity and phase transformation in shock wave propagation in nanocrystalline iron"; New Journal of Physics 16 (9), 093032. (2014)

- [He et al. 2017] - He, B. B.; Hu, B.; Yen, H. W.; Cheng, G.J.; Wang, Z.K.; Luo, H.W. and Huang, M.X. "High dislocation density-induced large ductility in deformed and partitioned steels"; Science 357 (6355), pp 1029-1032. (2017)

- [Kadau et al. 2002] - Kadau, K.; Germann T. C.; Lomdahi P. S. and Holian B. L. "Microscopic view of structural phase transitions induced by shock waves" Science 296 (5573), pp. 1681-1684. (2002)

- [Kadau et al. 2005] - Kadau, K.; Germann T. C.; Lomdahi P. S. and Holian B. L. "Atomistic simulations of shock-induced transformations and their orientation dependence in BCC Fe single crystals"; Physical Review B 72 (6), 064120. (2005)

- [Kanel et al. 2016] -/Kanel, G. i.; Zaretsky, E.; Razorenov, S.; Ashitkov, S.I. and Fortov, V.E. "Unusual plasticity and strength of metals at the ultra-short load durations"; Physics of Our Days (Uspekhi) 60 (5), pp. 490-508. (2016)

- [Khara et al. 2017] - Khara, Galvin S., Samuel T. Murphy, and Dorothy M. Duffy. "Dislocation loop formation by swift heavy ion irradiation of metals." Journal of Physics: Condensed Matter 29 (28), 285303 (2017)

- [Kittel 2004] - Kittel, C. "Introduction to Solid State Physics"; 8 edition, John Wiley \& Sons, ISBN 978-0-471-41526-8. (2004) 
- [Korchuganov et al 2015] - Korchuganov, A.V., et al. "Generation of shock waves in iron under irradiation." Nuclear Instruments and Methods in Physics Research Section B: Beam Interactions with Materials and Atoms, 352, pp.39-42. (2015)

- [Larsen et al. 2016] - Larsen, P. M.; Schmidt, S. and Schiøtz, J. "Robust structural identification via polyhedral template matching"; Modelling and Simulation in Materials Science and Engineering 24 (5), 055007. (2016)

- [Lehtinen et al. 2018] Lehtinen, A.; Laurson, L.; Granberg, F.; Nordlund, K. and Alava, M.J. "Effects of precipitates and dislocation loops on the yield stress of irradiated iron." Scientific Reports 8.1, 6914. (2018)

- [Marian et al. 2002] - Marian, J.; Wirth, B. D. and Perlado, J. M. “Mechanism of formation and growth of $<100>$ interstitial loops in ferritic materials"; Physical Review Letters 88, 255507.(2002)

- [Masters 1963] - Masters, B. C. "Dislocation loops in irradiated iron"; Nature 200, pp 254. (1963)

- [Masters 1965] - Masters, B. C. "Dislocation loops in irradiated iron"; The Philosophical Magazine 11 (113), pp 881-893. (1965)

- [Mendelev et al. 2003] - Mendelev, M.; Han, S.; Srolovitz, D.J.; Ackland, G.J.; Sun, D.Y. and Asta, M. "Development of new interatomic potentials appropriate for crystalline and liquid iron." The Philosophical Magazine 83 (35), pp. 3977-3994. (2003)

- [Nordlund et al. 1999] - Nordlund, K.; Keinonen, J.; Ghaly, M. and Averback, R. S. "Coherent displacement of atoms during ion irradiation"; Nature 398 (6722), pp. 49-51. (1999)

- [Nordlund et al. 2018a] - Nordlund, K. et al. "Primary radiation damage: A review of current understanding and models"; Journal of Nuclear Materials 512, pp. 450-479. (2018)

- [Nordlund et al. 2018b] - Nordlund, K. et al. "Improving atomic displacement and replacement calculations with physically realistic damage models"; Nature Communications 9, 1084. (2018)

- [Papaleo et al. 2015]- Papaleo, R. M.; Thomaz, R.; Gutierres, L. I.; De Menezes, V.M.; Severin, D.; Trautmann, C.; Tramontina, D.; Bringa, E.M. and Grande, P.L. "Confinement effects of ion tracks in ultrathin polymer films"; Physical Review Letters 114 (11), 118302. (2015)

- [Peng et al. 2018] -Peng, Q.; Meng, F.; Yang, Y.; Lu, C.; Deng, H.; Wang, L.; De, S. and Gao, F. "Shockwave generates $<100>$ dislocation loops in BCC iron"; Nature Communications 9 (1), 4880. (2018)

- [Pepperhoff 2001] - Pepperhoff, W.; Acet, M.; et al. "Constitution and Magnetism of Iron and its Alloys" Springer, Berlin. (2001).

[Plimpton 1995] - Plimpton, S. "Fast parallel algorithms for short-range molecular dynamics"; Journal of Computational Physics 117, pp. 1-19. (1995)

- [Proville et al. 2012] - Proville, L.; Rodney, D. and Marinica, M. "Quantum effect on the thermally activated glide of dislocation"; Nature Materials 11, pp 845-849. (2012) 
- [Sand et al. 2014] - Sand, A. E.; Nordlund, K. and Dudarev, S. L. "Radiation damage production in massive cascades initiated by fusion neutrons in tungsten"; Journal of Nuclear Materials 455 (1-3), pp. 207-211. (2014)

- [Schäublin et al. 2017] - Schäublin, R.; Décamps, B.; Prokhodtseva, A. and Löffler, J.F. "On the origin of primary $1 / 2 \mathrm{a}_{0}$ and $\mathrm{a}_{0}$ loops in irradiated $\mathrm{Fe}(\mathrm{Cr})$ alloys"; Acta Materialia 133, pp 427-439. (2017)

- [Smith et al. 2011] - Smith, R. F.; Eggert, J. H.; Rudd, R. E.; Swift, D.C.; Bolme, C.A. and Collins, G.W. "High strain-rate plastic flow in Al and Fe"; Journal of Applied Physics 110 (12), 123515. (2011)

- [Stoller 2012] - Stoller, R. E.; (ed. by) Konings, R. J. M. "Primary Radiation Damage Formation"; Volume 1, Elsevier, Amsterdam, p. 293-332. (2012)

- [Stoller 2016] - Stoller, R. E.; et al. "Impact of short-range forces on defect production from high-energy collisions"; Journal of chemical theory and computation 12(6), pp. 2871-2879. (2016)

- [Stukowski 2010] - Stukowski, A. "Visualization and analysis of atomistic simulation data with OVITO: The open visualization tool"; Modelling and Simulation in Materials Science and Engineering 18 (1), 015012.(2010)

- [Stukowski et al. 2012] - Stukowski, A.; Bulatov, V. V. and Arsenlis, A. "Automated identification and indexing of dislocations in crystal interfaces"; Modelling and Simulation in Materials Science and Engineering 20 (8), 085007. (2012)

- [Wang et al. 2019a] - Wang, X.; Gao, N.; Wang, Y.; Liu, H.; Shu, G.; Li, C.; Xu, B. and Liu, W. "Molecular dynamics study on the Burgers vector transition of nanometric dislocation loops induced by cascade in BCC-iron"; Journal of Nuclear Materials 519, pp. 322-331. (2019)

- [Wang et al. 2019b] - Wang, X.; Gao, N.; Wang, Y.; Wu, X.; Shu, G.; Li, C.; Li, Q.; $\mathrm{Xu}, \mathrm{B}$. and Liu, W. "Formation of $<100>$ dislocation loop in BCC-Fe via the ternary loop reaction"; Scripta Materialia 162, pp. 204-208. (2019)

- [Xu et al. 2013a] Xu, H.; Stoller, R. E. and Osetsky, Y. T. "Cascade defect evolution processes: Comparison of atomistic methods"; Journal of Nuclear Materials 443 (1-3), pp. 66-70. (2013)

- $\quad$ Xu et al. 2013b] - Xu, H.; Stoller, R. E.; Osetsky, Y. T. and Terentyev D. "Solving the puzzle of $<100>$ interstitial loop formation in BCC iron"; Physical Review Letters 110 (26), 265503. (2013)

- $\quad$ YYao et al, 2010] - Yao, Z.; Jenkins, M. L.; Hernández-Mayoral, M. and Kirk, M.A. "The temperature dependence of heavy-ion damage in iron: a microstructural transition at elevated temperatures"; The Philosophical Magazine 90 (35-36), pp 4623-4634. (2010)

- [Zel'Dovich et al. 1967] - Zel'Dovich, Ya. B.; Raizer and Yu. P. "Physics of shock waves and high-temperature hydrodynamic phenomena"; Academic Press, New York. (1967)

- [Ziegler et al. 1985] - Ziegler, J. and Biersack, J. "The stopping and range of ions in matter"; Treatise on heavy-ion science, Springer, Boston, pp. 93-129. (1985) 